\title{
Análise eletromiográfica de músculos do complexo do ombro durante exercícios de rotação externa com faixa elástica
}

\author{
Electromyographic analysis of muscles of the shoulder complex during external rotation \\ exercises with elastic band
}

\begin{abstract}
Análisis electromiográfico de los músculos del complejo del hombro durante ejercicios de rotación externa con banda elástica
\end{abstract}

Cássio Broilo', Rodrigo Costa Schuster ${ }^{2}$, William Dhein ${ }^{3}$

RESUMO | Na artrocinemática do ombro, o manguito rotador atua estabilizando a cabeça umeral em contato com a cavidade glenoidal, enquanto realiza a translação inferior para contrabalancear a força rotacional promovida principalmente pelo deltoide. Exercícios de rotação externa (RE) vêm sendo utilizados na reabilitação de pacientes com disfunções no complexo do ombro buscando restaurar a artrocinemática. Porém, poucos estudos abordam a utilização da faixa elástica e a avaliação dos músculos da cintura escapular, determinantes para uma cinemática adequada. O objetivo deste estudo é comparar a atividade eletromiográfica (EMG) de músculos do complexo do ombro durante exercícios de rotação externa com faixa elástica. Participaram 11 sujeitos do sexo masculino que foram avaliados durante os movimentos de (1) RE em ortostase; (2) RE com abdução de ombro; (3) RE em decúbito lateral (DL). Os músculos avaliados pela EMG foram: (1) trapézio superior (TS); (2) deltoide médio (DM); (3) deltoide posterior (DP); e (4) infraespinal (IN). A resistência foi determinada por uma faixa elástica cinza calibrada com carga de 5\% do peso corporal. A análise dos dados foi realizada no software Biomec-SAS e as estatísticas foram calculadas por meio da Anova de medidas repetidas no software SPSS v20.0. Pôde-se constatar que os músculos TS e DM obtiveram maior atividade EMG durante o exercício de RE com abdução de ombro, o músculo DP durante a RE com abdução de ombro e RE em decúbito lateral, enquanto o IN a obteve durante a RE em ortostase e RE em decúbito lateral.

Descritores | Ombro; Eletromiografia; Manguito Rotador; Terapia por Exercício.

ABSTRACT I In shoulder arthrokinematics, the rotator cuff acts on a lower base to counterbalance a glenoid cavity, while performing a lower translation to counterbalance a rotational force mostly provided by the deltoid. External rotation (ER) exercises have been used in the rehabilitation of patients with shoulder joint dysfunction, with the aim of restoring arthrokinematics. Few studies approached the use of the elastic band and the evaluation of the shoulder girdle muscles, essential for suitable kinematics. The objective of this study was to compare the electromyographic activity (EMG) of the muscles from the shoulder complex during external rotation exercises with elastic band. A total of 11 male subjects were evaluated during (1) ER in orthostasis; (2) ER with shoulder abduction; (3) ER with lateral decubitus. Themuscles assessed by theEMG were: (a) upper trapezius(UT); (b) middle deltoid (MD); (c) posterior deltoid (PD) and (d) infraspinatus (IS). Resistance was made with a gray elastic band calibrated with a load of $5 \%$ body weight. Data analysis was performed using the application software BIOMEC-SAS and statistics for the repeated measures using ANOVA in SPSS version 20.0. The UT and MD muscles were found to have increased EMG activity during ER exercise with shoulder abduction, as well as the PD muscle during 
ER with shoulder abduction and ER in lateral decubitus, whereas IS had increased EMG activity during an ER in orthostasis and ER in lateral decubitus.

Keywords I Shoulder; Electromyography; Rotator Cuff; Exercise Therapy.

RESUMEN | En la artrocinemática del hombro, el manguito rotador actúa estabilizando la cabeza umeral en contacto a la cavidad glenoidal, mientras realiza la traslación inferior para contrarrestar la fuerza rotacional promovida principalmente por el deltoides. Los ejercicios de rotación externa (RE) se han utilizado en la rehabilitación de pacientes con disfunciones en el complejo del hombro buscando restaurar la artrocinemática. Pocos estudios abordan la utilización de la banda elástica y la evaluación de los músculos de la cintura escapular, determinantes para una cinemática adecuada. El objetivo de este estudio es comparar la actividad electromiográfica (EMG) de los músculos del complejo del hombro durante ejercicios de rotación externa con banda elástica. Participaron 11 sujetos, varones, que fueron evaluados durante el movimiento de (1) RE en ortostasis; (2) RE con abducción de hombro; y (3) RE en decúbito lateral (DL). Los músculos evaluados por la EMG fueron: (a) trapecio superior (TS); (b) deltoides central (DC); (c) deltoides posterior (DP) e (d) infraespinoso (IN). La resistencia se determinó mediante una banda elástica gris calibrada con carga del $5 \%$ del peso corporal. El análisis de los datos se realizó con la utilización del software BIOMEC-SAS, y las estadísticas por medio de la Anova de medidas repetidas en el software SPSS v20.0. Se pudo constatar que los músculos TS y DC obtuvieron mayor actividad EMG durante el ejercicio de RE con abducción de hombro, el músculo DP durante la RE con abducción de hombro y RE en decúbito lateral, mientras que el IN durante la RE en ortostasis y en decúbito lateral.

Palabras clave I Hombro; Electromiografía; Manguito de los Rotadores; Terapia por Ejercicio.

\section{INTRODUÇÃO}

$\mathrm{Na}$ artrocinemática do ombro considerada normal, os músculos do manguito rotador (MR) atuam em conjunto, estabilizando a cabeça umeral em contato com a cavidade glenoidal ${ }^{1-3}$, enquanto deprimem a cabeça do úmero, evitando um impacto subacromial durante os movimentos de elevação do membro superior ${ }^{2-6}$. Além disso, a demanda dos músculos escapulotorácicos contribui para uma efetiva função do complexo do ombro $^{7-10}$.

Durante programas de prevenção ou de reabilitação do manguito rotador, os exercícios de rotação externa (RE) são geralmente usados com a intenção de melhorar a capacidade de estabilização e recrutamento do MR para restaurar o equilíbrio funcional e a artrocinemática do complexo do ombro. $\mathrm{Na}$ literatura, os exercícios de $R E$ vêm sendo investigados através da avaliação eletromiográfica (EMG) $)^{2,3,11-13}$. Apesar de a literatura apresentar diversos estudos avaliando o exercício de $\mathrm{RE}$, ainda assim, estes apresentam limitações. Pode-se citar que foram desconsideradas as influências dos músculos da cintura escapular, como trapézio superior, médio, inferior e serrátil anterior, os quais são determinantes para uma cinemática adequada. Ademais, cabe salientar que pacientes com síndrome do impacto do ombro (SIO) apresentam atividade eletromiográfica reduzida do músculo serrátil anterior, atividade retardada do trapézio médio e trapézio inferior, além de uma maior ativação do músculo trapézio superior ${ }^{14-19}$ e peitoral menor quando comparados a indivíduos saudáveis ${ }^{20}, \mathrm{o}$ que sustenta a importância da avaliação EMG desses músculos.

Outro aspecto relevante é que os estudos que avaliaram a atividade EMG durante exercícios de RE utilizam normalmente como carga externa o halter ${ }^{11-13}$ ou realizaram os exercícios de forma isométrica ${ }^{2,3}$. Entretanto, na fisioterapia os exercícios de rotação externa são comumente prescritos utilizando faixas elásticas ${ }^{21}$ como carga externa. Além de apresentar um baixo custo, as faixas são de fácil manuseio e apresentam ajustes individualizados, de modo que o fisioterapeuta pode variar, por exemplo, o ponto de fixação, a direção da aplicação da resistência e a tensão/resistência da faixa por meio da variação do comprimento ou das diferentes colorações ${ }^{21,22}$. Esses ajustes estão ligados diretamente à progressão da carga durante a evolução da prevenção/reabilitação ${ }^{8}$. Logo, cabe investigar as sobrecargas musculares durante variações de diferentes exercícios de RE de ombro.

Partindo do pressuposto de que exercícios de RE com faixa elástica como carga externa são importantes na reabilitação do ombro, o presente estudo tem como objetivo comparar a atividade EMG de músculos do complexo do ombro durante exercícios de RE com faixas elásticas. 


\section{METODOLOGIA}

\section{Delineamento do estudo e amostra}

Trata-se de um estudo de caráter quantitativo do tipo observacional e transversal, cuja amostra foi aleatória e composta por 11 sujeitos (idade média: 25,2 $\pm 3,7$ anos; peso médio: $76,6 \pm 7,7 \mathrm{~kg}$; altura média: $175,2 \pm 6,4 \mathrm{~cm}$ ) do sexo masculino com dominância do membro superior direito que apresentam amplitude de movimento (ADM) de pelo menos $30^{\circ}$ de rotação externa de ombro e força muscular preservada (grau 5). Os critérios de exclusão foram: (1) histórico de lesão musculotendínea no complexo do ombro; (2) fratura no complexo articular do ombro; (3) luxação ou subluxação; (4) disfunção cervical irradiada para membro superior. O cálculo amostral foi realizado no software $G^{*}$ Power 3.1.9.2, adotando os seguintes critérios: tamanho de efeito de 0,5 ; probabilidade de erro de $5 \%$; poder estatístico de 0,8 ; correlação entre as medições de 0,5 ; e assumindo esfericidade $(\mathrm{E}=1)$ para a família de testes estatísticos Anova para medidas repetidas.

\section{Procedimentos}

Todas as coletas foram realizadas por dois avaliadores e agendadas conforme disponibilidade dos participantes. Previamente às coletas, cada sujeito assinou o Termo de Consentimento Livre e Esclarecido e realizou uma avaliação de caracterização, contendo dados como: idade, peso e estatura. Inicialmente, o indivíduo foi submetido à realização de tricotomia e limpeza da pele com álcool nas regiões de colocação dos eletrodos. As variáveis eletromiográficas foram obtidas por meio do EMG Miotec de quatro canais dotados de um conversor A/D de 32bit conectado a um notebook da marca Lenovo. Foi utilizado o software MiotecSuite com uma taxa de amostragem de $2.000 \mathrm{~Hz}$.

Após a preparação da pele foi feita a colocação dos eletrodos de superfície $(\mathrm{Ag} / \mathrm{AgCl})$, conforme recomendações da literatura ${ }^{23-25}$ na configuração bipolar para os músculos trapézio superior (TS), deltoide médio (DM), deltoide posterior (DP) e infraespinal (IN), enquanto o eletrodo de referência foi posicionado na clavícula do indivíduo (Figura 1).

Após a colocação dos eletrodos, os indivíduos foram orientados a sentar-se em uma cadeira com apoio fixo para a realização das contrações isométricas voluntárias máximas (CIVM) para cada músculo avaliado neste estudo $^{23-25}$. A coleta da CIVM foi randomizada e consistiu em dois CIVM para cada músculo, mantendo a contração por cinco segundos e com intervalo de três minutos entre cada repetição.
A CIVM para o músculo deltoide posterior foi realizada com abdução de ombro e flexão de cotovelos a $90^{\circ}$, com rotação medial de $30^{\circ}$. O indivíduo tentava realizar o movimento de abdução, extensão e rotação externa contra a resistência manual do examinador. A CIVM para o músculo deltoide médio foi realizada com o membro superior do indivíduo mantidos a $90^{\circ}$ de abdução e com flexão de cotovelo, sendo que o examinador aplicou resistência na região distal do braço no sentido de adução de ombro. A CIVM do músculo trapézio superior foi realizado através da elevação dos ombros e inclinação da cabeça para o mesmo lado, onde uma faixa era posicionada sobre o ombro, impedindo a elevação pois o examinador mantinha suas mãos na cabeça impedindo a inclinação. A CIVM do infraespinal foi testada com o membro superior junto ao corpo e o cotovelo em flexão a $90^{\circ}$. O indivíduo realizava o movimento no sentido de rotação externa e o examinador fazia resistência no nível do cotovelo ${ }^{23-25}$.

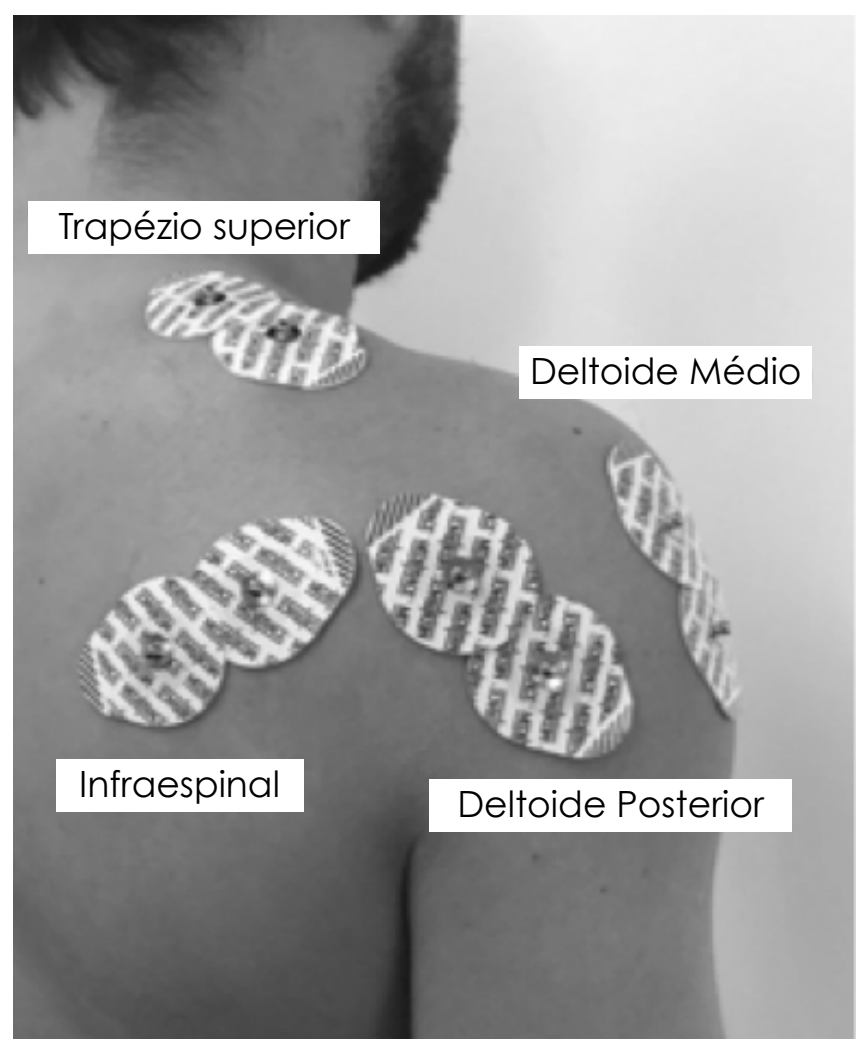

Figura 1. Posicionamento dos eletrodos de superfície para os músculos TS, DM, IE e DP

A seguir foram demonstrados e executados os movimentos pelos indivíduos, os quais consistem em: (A) rotação externa em ortostase com flexão de cotovelo a $90^{\circ}$; (B) rotação externa com abdução de ombro e flexão de cotovelo a $90^{\circ}$; (C) rotação externa em decúbito lateral (DL) e flexão de cotovelo a $90^{\circ}$ (Figura 2). 

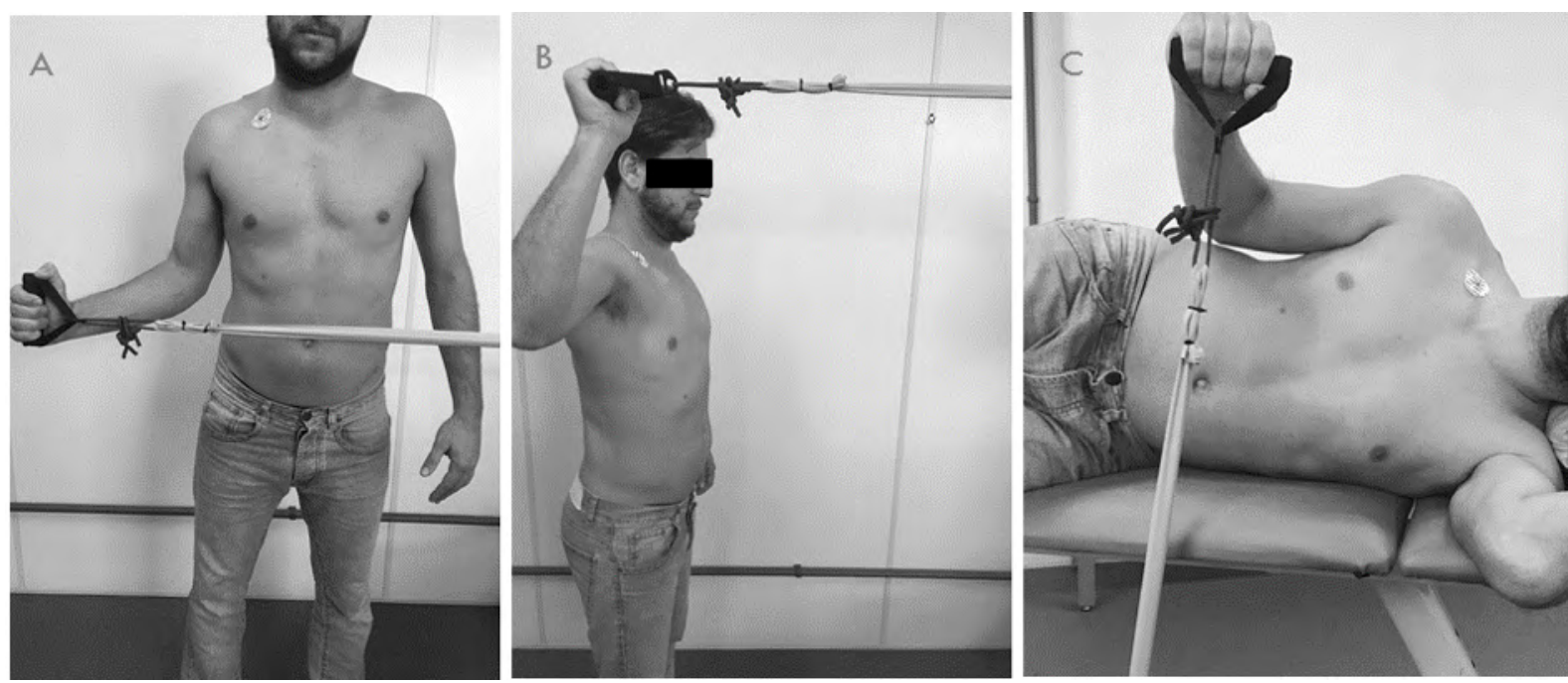

Figura 2. Movimentos de rotação externa avaliados com faixa elástica

Os participantes foram alinhados a webcam do computador e realizaram cinco repetições de cada movimento numa velocidade autosselecionada. Em todos os casos, foi utilizado uma resistência fornecida por uma faixa elástica cinza (Theraband) de $60 \mathrm{~cm}$ com a deformação calculada previamente pelos pesquisadores através de um procedimento de calibração da literatura ${ }^{21}$. $\mathrm{O}$ indivíduo teve que atingir uma resistência máxima de aproximadamente $5 \%$ do peso corporal no final de sua amplitude de movimento ${ }^{26}$ proveniente da calibração. Após a realização dos movimentos, foram desconectados os cabos e eletrodos, realizada a limpeza da pele do indivíduo e a participação na pesquisa estava encerrada.

\section{Análise dos dados}

Os sinais eletromiográficos foram analisados utilizando o software Biomec SAS ${ }^{27}$. Inicialmente os sinais coletados durante as CIVM e os movimentos de rotação foram submetidos a um procedimento de filtragem digital, utilizando um filtro Butterworth passa-banda de quarta ordem, com banda de frequência entre $20 \mathrm{~Hz}$ e $400 \mathrm{~Hz}$. Após o procedimento de filtragem foram descartados a primeira e a última repetição, restando três sinais centrais com dados cinemáticos obtidos pela webcam. Já nas CIVMs foi utilizado o valor de pico do envelope root mean square (RMS) de cada músculo coletado. $\mathrm{O}$ sinal eletromiográfico foi processado no domínio do tempo a partir do cálculo de envelope RMS com a função de janela de Hamming de um segundo. $\mathrm{O}$ valor RMS de cada músculo estudado foi normalizado pela razão entre o valor RMS e o valor de pico da CIVM.

\section{Análise estatística}

A análise estatística foi realizada no software SPSS 20.0. Inicialmente foram verificadas as normalidades dos dados por meio do teste de Shapiro-Wilk. As comparações dos dados eletromiográficos para cada um dos músculos foram realizadas através da Anova de medidas repetidas, sendo considerado o fator exercício com três níveis: (1) rotação externa em ortostase com flexão de cotovelo a $90^{\circ}$; (2) rotação externa com abdução de ombro e flexão de cotovelo a 90\%; e (3) rotação externa em decúbito lateral e flexão de cotovelo a $90^{\circ}$. Os resultados estatísticos serão reportados pelo valor da razão de $\mathrm{F}$ (Anova), nível de significância (valor de p) e tamanho de efeito eta ${ }^{2}$ (eta quadrado). Em caso de diferenças nas comparações foi utilizado o teste post hoc de Bonferroni, adotando um nível de significância de $\alpha<0,05$.

\section{RESULTADOS}

Considerando a atividade eletromiográfica de músculos do complexo do ombro durante as variações dos exercícios de rotação externa com faixa elástica (Figura 3), podese constatar, com base na Anova de medidas repetidas, diferenças para o fator "movimento" nos músculos TS $\left(\mathrm{F}=23,582 ; \mathrm{p}<0,0001 ; \mathrm{eta}^{2}=0,702\right), \mathrm{DM}(\mathrm{F}=20,174$; $\mathrm{p}<0,0001 ;$ eta $\left.^{2}=0,669\right), D P(F=15,343 ; \mathrm{p}<0,0001$; eta $\left.^{2}=0,605\right)$ e IN $\left(F=4,783 ; p=0,020 ;\right.$ eta $\left.^{2}=0,324\right)$.

Com base no teste post hoc de Bonferroni, pode-se observar que os músculos TS e DM obtiveram maior atividade EMG durante o exercício de RE com abdução de ombro, quando comparados a RE em ortostase 
$(\mathrm{p}=0,001 ; \mathrm{p}=0,015)$ e em DL $(\mathrm{p}=0,005 ; \mathrm{p}=0,001)$. Também se observou maior atividade EMG durante a RE em DL quando comparado a RE em ortostase $(\mathrm{p}=0,033 ; \mathrm{p}=0,006)$.

Considerando o músculo DP pode-se constar maiores magnitudes EMG durante os exercícios de RE com abdução de ombro e em DL, quando comparados a em ortostase $(p=0,003 ; p<0,0001)$. Não se observou diferença quando comparados os exercícios de RE com abdução de ombro e em DL ( $\mathrm{p}=0,941)$.

Considerando o músculo IN, pode-se constatar maiores magnitudes EMG durante o exercício de RE em DL quando comparados a RE com abdução de ombro $(p=0,05)$. Não se observou diferença quando comparados os exercícios de RE em ortostase e em DL ( $p=0,395)$ com $\mathrm{RE}$ com abdução de ombro ( $\mathrm{p}=0,435)$.

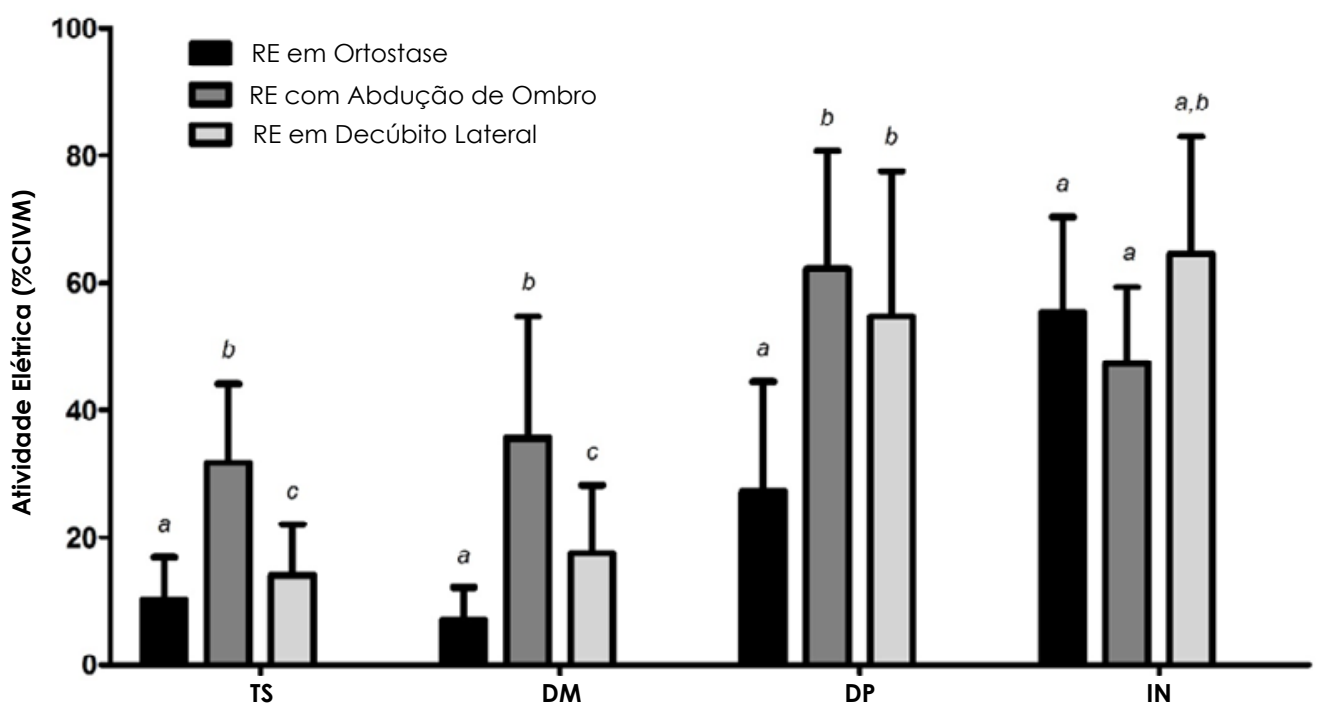

Figura 3. Atividade eletromiográfica durante variações de exercícios de rotação externa com faixa elástica

TS: trapézio superior; DM: deltoide médio; DP: deltoide posterior; IN: infraespinal (IN).

Letras diferentes indicam diferença estatística.

\section{DISCUSSÃO}

O objetivo deste estudo foi comparar a atividade eletromiográfica de músculos do complexo do ombro durante exercícios de rotação externa com faixa elástica. Pôde-se constatar que os músculos TS e DM obtiveram maior atividade EMG durante o exercício de RE com abdução de ombro, o músculo DP durante a RE com abdução de ombro e RE em DL, enquanto o IN durante a RE em ortostase e em DL.

Durante o exercício de RE com abdução de ombro, os músculos TS e DM apresentaram maior atividade EMG, quando comparados a em ortostase e em DL. Esses resultados podem ter ocorrido devido à maior exigência de estabilização escapular do TS para manter a escápula elevada e em rotação superior ${ }^{9,15,19}$, juntamente com grande solicitação do DM na estabilização glenoumeral de abdução em isometria para realizar a $\mathrm{RE}$ através dos músculos $\mathrm{DP}$ e IN ${ }^{11}$. Quando verificamos a $\mathrm{RE}$ em ortostase, constatamos menor atividade EMG do TS, pois não há necessidade de manter a escápula elevada e em rotação superior para auxiliar na abdução da glenoumeral, havendo menor solicitação também do DM, pois o ombro se mantém em posição neutra. Desse modo, o recrutamento tanto do TS quanto do DM fica menor.

Quando comparadas as situações de RE em ortostase com as de RE em DL, especulamos que os indivíduos tendem a realizar uma abdução da articulação glenoumeral, o que pode afetar diretamente a atividade EMG do DM. Normalmente, essa abdução é controlada com a utilização de um coxim ou de uma toalha apoiada no cotovelo $^{3,8}$. O uso do coxim ou da toalha tem o objetivo de diminuir a contribuição do DM e DP na translação superior da cabeça umeral, o que já foi evidenciado com a $\mathrm{EMG}^{3,8}$. Entretanto, não obtivemos dados cinemáticos que comprovem a hipótese de os indivíduos realizarem a abdução de ombro.

Reinold et al. ${ }^{11}$ avaliaram diversos músculos durante variações dos exercícios de rotação externa com halter. $\mathrm{O}$ músculo DM obteve a sua maior atividade EMG durante o exercício de RE na posição de prono e extensão horizontal, 
seguido da RE com abdução de ombro. Mesmo que não tenhamos avaliado o movimento em pronação, podemos assumir que os resultados desta pesquisa são idênticos aos avaliados no estudo dos autores, que obtiveram as maiores magnitudes de DM durante a RE com abdução, seguido por RE em DL e em ortostase.

Considerando os resultados encontrados na literatura para o músculo TS durante a $\mathrm{RE}^{28}$, foram avaliadas as diferentes porções do trapézio e deltoide posterior em variações de exercícios. As comparações de timing/tempo de ativação (extensão de ombros em decúbito ventral, RE em DL com coxim, extensão horizontal em DL e extensão horizontal em prono) constataram que o TS tem maior ativação em um momento posterior ao do DP e que, portanto, acaba sendo utilizado na parte final do movimento. Possivelmente a maior ativação adquirida pelo TS em DL quando comparado à posição em ortostase se justifica pela influência da força gravitacional sobre os segmentos, o que acaba exigindo mais do TS e do DP para a realização do movimento.

Como não houve diferença estatística no post hoc para o músculo DP entre as situações RE com abdução e em DL, considera-se que o músculo DP obteve suas maiores atividades EMG em ambas as atividades. Considerando esse aspecto estatístico, o IN obteve suas maiores atividades durante a RE em ortostase e em DL. Os resultados referentes ao DP foram semelhantes aos encontrados na literatura ${ }^{12,11}$. O músculo DP obteve as menores atividades EMG durante a RE em sedestação com flexão de cotovelo e não houve diferenças nas atividades EMG entre o exercício em decúbito ventral, em DL e sentado com abdução horizontal ${ }^{12}$. Outro estudo também não encontrou diferenças entre a situação RE com abdução e DL, mas obteve a maior atividade EMG do DP em decúbito ventral com abdução horizontal ${ }^{11}$. Dessa forma, demonstra que a contribuição do DP parece ser maior nos movimentos que envolvem a abdução horizontal, inclusive estudos mencionam que o deltoide posterior pode contribuir como um adutor no movimento de RE na posição neutra, mantendo a adução com o uso do coxim ${ }^{2,3}$.

A atividade EMG encontrada no músculo IN durante o exercício de rotação externa em DL e na RE em ortostase também foi verificada na literatura ${ }^{11}$. Sua maior ativação foi no exercício de RE em DL e se diferenciou do exercício de RE em ortostase com a utilização de um rolo no cotovelo. No presente estudo, não houve diferença entre a situação RE em DL e em ortostase, provavelmente devido à utilização do rolo na literatura ${ }^{11}$. Em contrapartida, no presente estudo encontrou-se uma diferença entre a situação de RE em DL com RE com abdução. Especulamos esse resultado devido ao fato de as cargas externas utilizadas serem as faixas elásticas, as quais promovem uma alteração no vetor de força ao longo da amplitude de movimento, ao passo que nos halteres esse vetor segue a força gravitacional. Não encontrar diferença entre as situações em ortostase e em DL para o infraespinal pode estar relacionado também ao número amostral, visto que o tamanho de efeito do IN foi de 0,324 inferior ao estipulado no cálculo amostral.

Hintermeister et al. ${ }^{22}$ também constataram que o infraespinal obteve as maiores atividades EMG durante o movimento de RE em ortostase. Entretanto, os pesquisadores não avaliaram outras variações de $R E$. Outro estudo avaliou a ativação de IN, DM e DP durante a isometria de $\mathrm{RE}$ com $10 \%, 40 \%$ e $70 \%$ da repetição máxima $(\mathrm{RM})^{3}$, podendo-se observar menores atividades do DM e do DP, e maiores atividades do IN com uma carga de 40\% de RM, o que seria indicativo de uma carga ideal para o recrutamento do IN. No presente estudo, optamos por utilizar o percentual do peso corporal, o que equivaleu aproximadamente a $3,8 \mathrm{~kg}$ de resistência na faixa elástica.

Considerando os resultados do estudo, conclui-se que há uma justificativa cientifica para o uso dos exercícios investigados em diferentes programas de prevenção e reabilitação ${ }^{8,28}$. O movimento de RE com abdução da glenoumeral pode ser utilizado na fase final de uma reabilitação onde o objetivo seja recrutar os músculos TS, DM e DP, os quais normalmente estão alterados na SIO na fase inicial. Já o músculo IN apresenta hipoatividade na fase inicial com SIO. Sendo assim, indica-se a aplicabilidade dos exercícios de RE com flexão de cotovelo ou em DL. Dessa forma, os objetivos primários de um programa de exercícios devem focar em ativar os músculos do manguito rotador e escapulares em movimentos que não gerem contato subacromial ou que ponham em estresse músculos hiperativados ${ }^{8}$. Chama a atenção que, quando observamos o DP e o IN durante os movimentos de RE com abdução e em DL, notamos uma inversão das atividades. Ou seja, parece que, se buscamos otimizar o IN e diminuir a influência indesejada do DP e $\mathrm{DM}^{3,8}$, devemos realizar os exercícios em DL ou em ortostase.

Este estudo apresenta limitações e perspectivas futuras com base nos resultados encontrados. Citamos que a velocidade foi autosselecionada e a amplitude de movimento foi padronizada ao máximo para cada indivíduo, o que pode ter influenciado alguns resultados 
por causa de diferenças anatômicas. Entretanto, teve-se o cuidado de orientar os participantes com execuções controladas. No presente estudo, a carga externa foi fixada de acordo com 5\% do peso corporal do indivíduo, diferentemente de estudos ${ }^{2,3}$ que determinaram suas cargas em relação ao percentual de RM (10\%, 40\% e $70 \%)$ ou a cargas fixas ${ }^{12}$. Tal aspecto metodológico pode gerar resultados distintos quando comparado com outros estudos. Portanto, a definição da magnitude da carga externa é um aspecto importante e que deve ser levado em conta na prescrição de exercícios. Outra limitação deste estudo é que diversos músculos do complexo do ombro foram desconsiderados, pois os instrumentos de avaliação de EMG contavam com um número restrito de canais. Assim, músculos como trapézio médio, trapézio inferior, serrátil anterior e redondo menor, que estão relacionados com a SIO, precisam ser avaliados em estudos futuros. Os resultados encontrados neste estudo não se aplicam a indivíduos com patologias no ombro, pois não sabemos como as alterações EMG de pacientes com SIO influenciam na execução dos exercícios. Porém, mesmo assim, podemos pensar devidamente no uso dos exercícios avaliados como forma de prevenção de lesões no complexo do ombro. Destacamos que as ativações musculares podem mudar dependendo do tipo de resistência empregado (halter ou faixa elástica).

Desse modo, são notórios os resultados da presente pesquisa no âmbito da reabilitação, haja vista sua aplicabilidade terapêutica e já que outros estudos da literatura se limitam a investigar apenas cargas com halteres e sem individualização de acordo com os participantes. Entendemos que ainda existem lacunas a serem exploradas do ponto de vista de exercícios de RE nas diferentes fases do movimento (concêntrico e excêntrico) e no timing da ativação EMG, bem como faltam estudos que abordem a influência desses exercícios sobre pacientes com SIO.

\section{CONCLUSÃO}

Pôde-se constatar que os músculos trapézio superior e deltoide médio obtiveram maior atividade EMG durante o exercício de rotação externa com abdução de ombro, o músculo deltoide posterior durante a rotação externa com abdução de ombro e rotação externa em decúbito lateral, enquanto o músculo infraespinal a obteve durante a rotação externa em ortostase e rotação externa em decúbito lateral.

\section{REFERÊNCIAS}

1. Aboelmagd T, Rees J, Gwilym S. Rotator cuff tears: pathology and non-surgical management. Orthop Trauma. 2018;32(3):159-64. doi: 10.1016/j.mporth.2018.03.003

2. Clisby EF, Bitter NL, Sandow MJ, Jones MA, Magarey ME, Jaberzadeh S. Relative contributions of the infraspinatus and deltoid during external rotation in patients with symptomatic subacromial impingement. J Shoulder Elbow Surg. 2008;17(1):S87-S92. doi: 10.1016/j.jse.2007.05.019

3. Bitter NL, Clisby EF, Jones MA, Magarey ME, Jaberzadeh S, Sandow MJ. Relative contributions of infraspinatus and deltoid during external rotation in healthy shoulders. J Shoulder Elbow Surg. 2007;16(5):563-8. doi: 10.1016/j.jse.2006.11.007

4. de Oliveira FCL, Bouyer LJ, Ager AL, Roy JS. Electromyographic analysis of rotator cuff muscles in patients with rotator cuff tendinopathy: a systematic review. J Electromyogr Kinesiol. 2017;35:100-14. doi: 10.1016/j.jelekin.2017.06.002

5. Lewis J, McCreesh K, Roy JS, Ginn K. Rotator cuff tendinopathy: navigating the diagnosis-management conundrum. J Orthop Sports Phys Ther. 2015;45(11):923-37. doi: 10.2519/jospt.2015.5941

6. Reddy AS, Mohr KJ, Pink MM, Jobe FW. Electromyographic analysis of the deltoid and rotator cuff muscles in persons with subacromial impingement. J Shoulder Elbow Surg. 2000;9(6):519-23. doi: 10.1067/mse.2000.109410

7. Veado MAC, Flóra W. Reabilitação pós-cirúrgica do ombro. Rev Bras Ortop. 1994;29(9):661-4.

8. Ellenbecker TS, Cools A. Rehabilitation of shoulder impingement syndrome and rotator cuff injuries: an evidence-based review. $\mathrm{Br}$ J Sports Med. 2010;44(5):319-27. doi: 10.1136/bjsm.2009.058875

9. Ludewig PM, Reynolds JF. The association of scapular kinematics and glenohumeral joint pathologies. J Orthop Sports Phys Ther. 2009;39(2):90-104. doi: 10.2519/jospt.2009.2808

10. Batista LP, Oliveira VA, Pirauá AR, Pitangui AR, Araújo RC. Atividade eletromiográfica dos músculos estabilizadores da escápula durante variações do exercício push up em indivíduos com e sem síndrome do impacto do ombro. Motricidade. 2013;9(3):70-81. doi: 10.6063/motricidade.9(3).769

11. Reinold MM, Wilk KE, Fleisig GS, Zheng N, Barrentine SW, Chmielewski T, et al. Electromyographic analysis of the rotator cuff and deltoid musculature during common shoulder external rotation exercises. J Orthop Sports Phys Ther. 2004;34(7):385-94. doi: 10.2519/jospt.2004.34.7.385

12. Kim J-W, Yoon J-Y, Kang M-H, Oh J-S. Selective activation of the infraspinatus during various shoulder external rotation exercises. J Phys Ther Sci. 2012;24(7):581-4. doi: 10.1589/jpts.24.581

13. Lim OB, Kim JA, Song SJ, Cynn HS, Yi CH. Effect of selective muscle training using visual EMG biofeedback on infraspinatus and posterior deltoid. J Hum Kinet. 2014;44(1):83-90. doi: 10.2478/hukin-2014-0113

14. Spall P, Ribeiro DC, Sole G. Electromyographic activity of shoulder girdle muscles in patients with symptomatic and asymptomatic rotator cuff tears: a systematic review and meta-analysis. PM R. 2016;8(9):894-906. doi: 10.1016/j.pmrj.2016.02.015

15. Ludewig PM, Cook TM. Alterations in shoulder kinematics and associated muscle activity in people with symptoms of shoulder impingement. Phys Ther. 2000;80(3):276-91. 
16. Lin J-J, Hanten WP, Olson SL, Roddey TS, Sherwood AM, Lim HK, et al. Functional activity characteristics of individuals with shoulder dysfunctions. J Electromyogr Kinesiol. 2005;15(6):576-86. doi: 10.1016/j.jelekin.2005.01.006

17. Diederichsen LP, Nørregaard J, Dyhre-Poulsen P, Winther A, Tufekovic G, Bandholm T, et al. The activity pattern of shoulder muscles in subjects with and without subacromial impingement. J Electromyogr Kinesiol. 2009;19(5):789-99. doi: 10.1016/j. jelekin.2008.08.006

18. Huang TS, Ou HL, Huang CY, Lin JJ. Specific kinematics and associated muscle activation in individuals with scapular dyskinesis. J Shoulder Elbow Surg. 2015;24(8):1227-34. doi: 10.1016/j.jse.2014.12.022

19. Phadke V, Camargo PR, Ludewig PM. Scapular and rotator cuff muscle activity during arm elevation: a review of normal function and alterations with shoulder impingement. Rev Bras Fisioter. 2009;13(1):1-9. doi: 10.1590/S1413-35552009005000012

20. Castelein B, Cagnie B, Parlevliet T, Cools A. Scapulothoracic muscle activity during elevation exercises measured with surface and fine wire EMG: a comparative study between patients with subacromial impingement syndrome and healthy controls. Man Ther. 2016;23:33-9. doi: 10.1016/j.math.2016.03.007

21. Loss JF, Koetz AP, Soares DP, Scarrone FF, Hennemann V, Sacharuk VZ. Quantificação da resistência oferecida por bandas elásticas. Rev Bras Cienc Esporte. 2002;24(1):61-72.

22. Hintermeister RA, Lange GW, Schultheis JM, Bey MJ, Hawkins RJ. Electromyographic activity and applied load during shoulder rehabilitation exercises using elastic resistance. Am J Sports Med. 1998;26(2):210-20. doi: 10.1177/03635465980260021001

23. Hermens HJ, Freriks B, Disselhorst-Klug C, Rau G. Development of recommendations for SEMG sensors and sensor placement procedures. J Electromyogr Kinesiol. 2000;10(5):361-74. doi: 10.1016/S1050-6411(00)00027-4

24. Selkowitz DM, Chaney C, Stuckey SJ, Vlad G. The effects of scapular taping on the surface electromyographic signal amplitude of shoulder girdle muscles during upper extremity elevation in individuals with suspected shoulder impingement syndrome. J Orthop Sports Phys Ther. 2007;37(11):694-702. doi: 10.2519/jospt.2007.2467

25. Ribeiro DC, de Castro MP, Sole G, Vicenzino B. The initial effects of a sustained glenohumeral postero-lateral glide during elevation on shoulder muscle activity: a repeated measures study on asymptomatic shoulders. Man Ther. 2016;22:101-8. doi: 10.1016/j.math.2015.10.014

26. de Toledo JM, Ribeiro DC, de Castro MP, Forte FC, Körbes TS, Rusch MW, et al. Comparison of shoulder resultant net moment between three different exercises and load conditions. Physiother Theory Pract. 2013;29(2):124-32. doi: 10.3109/09593985.2012.699606

27. Torre ML. Desenvolvimento de um software para processamento e análise de sinais biológicos utilizados em biomecânica [tese]. Porto Alegre: Universidade Federal do Rio Grande do Sul; 2013.

28. De Mey K, Cagnie B, Van De Velde A, Danneels L, Cools AM. Trapezius muscle timing during selected shoulder rehabilitation exercises. J Orthop Sports Phys Ther. 2009;39(10):743-52. doi: 10.2519/jospt.2009.3089 